\title{
Strategy for Load Balancing Task Assignment Based on Traffic Load
}

\author{
Xu Xudong ${ }^{1, a}$, Zhou Xueyang ${ }^{2, b}$ \\ ${ }^{1}$ Faulty of Information Technology, Beijing University of Technology, Beijing, China \\ ${ }^{2}$ Faulty of Information Technology, Beijing University of Technology, Beijing, China \\ a xuxudong@bjut.edu.cn, b463691225@qq.com
}

Keywords: traffic load; load balancing; workflow system; minimum variance; mobile internet

\begin{abstract}
Aiming at the problem that in the mobile Internet platform, the traffic load (that is, the time the task participant costs when arriving at the task execution site) has a great impact on load balancing of task participant and execution efficiency of task in the workflow system, this paper proposes a load balancing task assignment strategy considering the traffic load. On the basics of considering execution load of task, the strategy fully considers the traffic load due to the decentralization of the task execution site when calculating the load of task participant. Additionally to the variance of the load sequence constituted by loads of all the task participants as a standard, selecting the assignment scheme with the smallest variance of load sequence after task assignment in all assignment schemes. The application of this strategy can significantly improve the load balancing effect of task participant and the overall execution efficiency of the task in the workflow system under the mobile Internet platform.
\end{abstract}

\section{Introduction}

Workflow technology is one of the research hotspots in computer field at present. It attracts the attention of researchers and enterprises due to the compliance to the development trend of the flow-oriented management mode. Workflow management system (WfMS) is the system defining, establishing and implementing workflow. It is a special computer supported cooperative work (CSCW) [1]. The workflow management system mainly decomposes workflow task into multiple sub-task steps, to assign subtasks to various system roles as per certain assignment strategy; the task assignment strategy in the workflow system is one of the key orientations of researches on workflow technology.

The role-based task assignment method is introduced in workflow meta model of Workflow management coalition (WfMC) and most workflow management systems; i.e., it is the abstract role but not specific person is assigned as the performer of the task during the defining of workflow. The role based work task assignment method can reduce the variation on definition of work flow; however, a role may be corresponding to multiple specific task participation [2]. Therefore, a reasonable task assignment strategy is needed for workflow engine, to achieve task burden balance between multiple task participants corresponding to the same role, to improve overall work task efficiency.

Domestic and overseas conduct positive exploration on the research orientation of task assignment strategy; two main modes for task assignment strategies have been established at present: rigid assignment and dynamic assignment. During the workflow system in early stage, rigid assignment mode is taken as the main task assignment 
strategy, with the content that the specific performer of the work task of each procedure has been set during the flow definition process, i.e., before the flow instantiation [3]. This strategy can ensure the correct assignment result to certain extent; however, with the increase of business of workflow system, some disadvantages of rigid assignment have been exposed such as rigid, inflexible and impractical; besides, the rigid task assignment strategy has very low efficiency under the environment of large task load, cannot meet the business requirements. Due to multiple defects of rigidly assigned task assignment strategy, the dynamically assigned task assignment strategy has been widely applied to various workflow systems; dynamic assignment strategy conducts task assignment through the consideration to influencing factors and the evaluation on various conditions in a comprehensive and real-time way, including work assignment influencing factors such as load, work capability, etc. [3] The efficiency of dynamic assignment strategy is much higher than that of the rigidly assigned task assignment strategy, and is more flexible.

\section{Related Research Work}

Numerous researchers have conducted positive explorations on the researches on dynamic task assignment strategy.

Literature [2] proposes a task assignment strategy in support of load balance of task participants and empirical value, to establish relation between roles and task performers. On the basis of load prediction to task participant, this strategy takes comprehensive consideration on factors of task participants into consideration, such as the work load as well as accomplishment quality and interests to different types of works.

Literature [4] proposes a task and user property based workflow task assignment algorithm (WAMTU); through procedures such as role matching, skill matching, load and empirical value matching, this algorithm selects the optimal task participant of the new task.

Literature [5] proposes a dynamic task assignment work model S-DTAWM which gives priority to strategy. By utilizing the descriptive language of set theory, it defines contents, work relation and applicable algorithm of various components of S-DTAWM in a formalized way, which is mainly applied to business-oriented general dynamic task assignment system.

All the above researches have taken the influences of accepted task on load into consideration during the quantum calculation on the load of task participants; however, they only calculate the sum of the time needed for implementation of accepted tasks for participants, and the sum of the time is taken as the load value of corresponding task participants. However, in the mobile internet platform based workflow system, due to the scattered geographical locations for implementation of tasks, the traffic load of participants has significant influence on task completion efficiency. However, the above two task assignment algorithms fail to take traffic load into consideration; therefore, the two algorithms fail to have an ideal task assignment effect in mobile internet platform based workflow system.

Based on the above conclusion, this article proposes a traffic load based load balance task assignment strategy. By taking sufficient consideration to the traffic load of task 
participants during task assignment of the workflow system, this strategy takes the sum of task load and traffic load as the load quantized value of the task participant, and the load variance of all the task participants in the system after task assignment as the standard for task assignment, to ensure that the load variance after each assignment is the minimum, to improve the overall task assignment efficiency in the system.

\section{Task Assignment Strategies}

Basic Idea. Task assignment aims to assign according to the time needed for implementation of tasks, to ultimately achieve balance between all the task participants; i.e., the variance of the set composed by the load of all task participants is the least. Theoretically, the participant with the least load at present shall be selected for task assignment; however, in the workflow system involved in traffic load, the increase of task load is not simply sum of load, and the practical traffic load for task participants to arrive at the task location shall be taken into consideration; therefore, the simple selection on the participant of the least load may not achieve the purpose of load balance between all task participants.

Therefore, during task assignment, the strategy proposed in this article conducts a task assignment to all task participants, and then calculate the least time for each task participant to arrive at all the task implementation locations in the task list after path planning, which is then added with the time needed for implementation of all tasks, and the sum is taken as the load value of the task participant. Then calculate the variance of the set composed by the load of all the task participants, and select assignment task, to select the task participant with the least load set variance, and conduct task assignment. Related Definitions.

- Definition $1 \mathrm{U}$ is the set of task participants, which includes all the valid task participants in workflow system; $\mathrm{N}$ is the number of participants, participant $\mathrm{Ui}$ $\in \mathrm{U}, \mathrm{i}=1,2, \ldots, \mathrm{N}$.

- Definition $2 \mathrm{~J}$ is the set of the assigned tasks of participant $\mathrm{Ui}$; $\mathrm{K}$ is the number of the assigned tasks of the participant $\mathrm{Ui}$; $\mathrm{JT} \in \mathrm{J}$ is the set time of all tasks in $\mathrm{J}$; single task time $\mathrm{JTi} \in \mathrm{JT}, \mathrm{i}=1,2,3, \ldots, \mathrm{K}, \mathrm{JL} \in \mathrm{J}$ is the set of location information of all tasks in $\mathrm{J}$; single task location information $\mathrm{JLi} \in \mathrm{JL}, \mathrm{i}=1,2,3, \ldots, \mathrm{K}$.

- Definition $3 \mathrm{TL}()$ is the algorithm used to calculate the shortest path of traffic load, with parameters of the set of location information, and with the calculation result of the shortest time needed to traverse all the locations in the location information set.

- Definition 4 The task load of the participant Ui is WOL(Ui), with the following calculation formula:

$$
W O L\left(U_{i}\right)=\sum_{i=1}^{k} J T_{i}+T L(J L) .
$$

- Definition 5 WOLU is the set of the task participant load, which includes current load of all the participants, i.e.:

$$
W O L U=\left\{W O L\left(U_{i}\right) \mid i=1,2,3, \ldots, N\right\} .
$$


- Definition $6 \mathrm{D}()$ is variance calculation, with the parameter of the set of the load of task participants, and with the calculation result as the variance value of a group of data of the load set, i.e.:

$$
D(W O L U)=\sum_{i=1}^{N}\left(W O L\left(U_{i}\right)-\overline{W O L U}\right)^{2} .
$$

In which $\overline{W O L U}$ is the average value of WOLU, the set of the load of participants. Algorithm Description.

1) As for the task $\mathrm{j}$ to be assigned, acquire the expected task time jt, and the task implementation location $\mathrm{jl}$.

2) for $(\mathrm{i}=1 ; \mathrm{i}<=\mathrm{N} ; \mathrm{i}++)\{$

a) Calculate the load WOL(Ui') of the task participant Ui, who is assigned with the task $\mathrm{j}$, with the following calculating formula:

$$
W O L\left(U_{i}^{\prime}\right)=\sum_{i=1}^{K} J T_{i}+j t+T L(J L+j l) .
$$

b) Acquire the set of the load of task participants after assignment WOLUi' .

c) Calculate the variance $\mathrm{D}$ (WOLUi').

3) Define Um as the ultimately assigned task participant, $1 \leq \mathrm{m} \leq \mathrm{N}$, and:

$$
D\left(W O L U_{m}{ }^{\prime}\right)=\min _{1 \leq i \leq N} D\left(W O L U_{i}^{\prime}\right) .
$$

4) Assign task $\mathrm{j}$ to Um, and the algorithm ends.

\section{Performance Analysis}

Comparative Algorithm. In order to evaluate on the performance of the load balance task assignment strategy proposed in this article, multiple dynamic task assignment algorithms mentioned in the above are selected for comparison. In consideration that all the mentioned algorithms calculate the sum of the time needed for all the tasks in the task list of all the task participants, and select the participant with the least calculate result; therefore, a task assignment algorithm can be abstracted, as the comparative algorithm in the experiment; this algorithm traverses the task lists of all the task participants in the system when assigning new tasks, and adds the time needed in the task list, and the sum is taken as the load of the participant; finally, the participant with the least load is selected.

Experimental Scheme. Formulate the scheme for the performance comparison experiment: select 3 task participants in the experiment (ui,i=1,2,3), with the initial state of their task lists as blank. At the beginning of the experiment, 200 tasks are generated randomly, and the task location and needed time of each task is generated randomly within reasonable scope. Then assign the 200 tasks to 3 task participants with the algorithm proposed in this article and the comparative algorithm, respectively, and then record the load value and the load variance of the 3 task participants after the assignment. Finally, make comparison on the recorded data between the two algorithms, and conduct analysis and comparison between results.

Result and Analysis. 


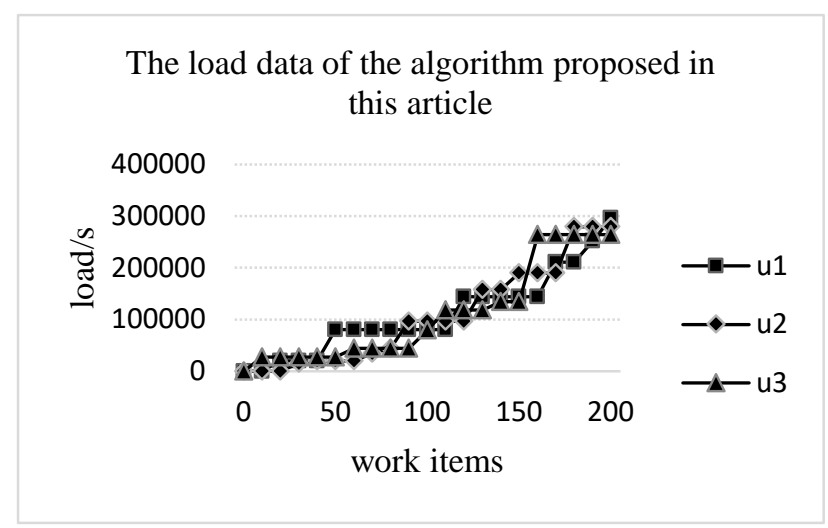

Fig. 1 The Work Load of the Algorithm Proposed in This Article.

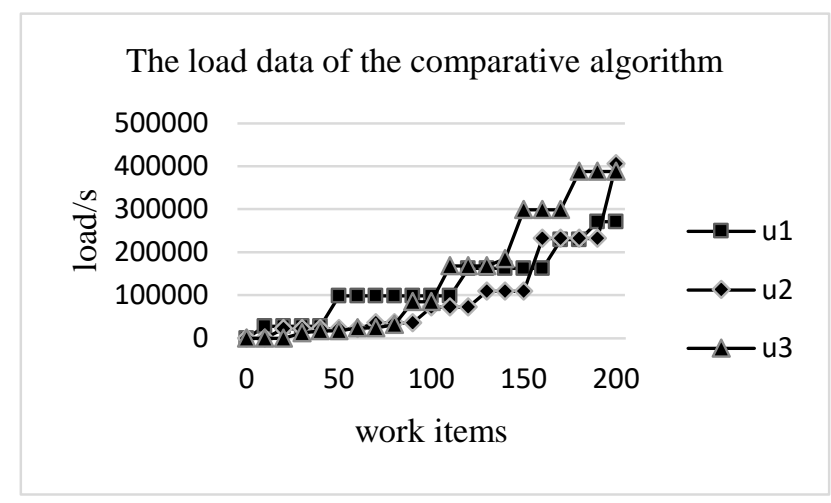

Fig. 2 The Work Load of the Comparative Algorithm

It can be found from the comparison on the data between Fig. 1 and Fig. 2 that the algorithm proposed in this article significantly reduces the separation degree between the task participants. Compared with the comparative algorithm, the algorithm proposed in this article achieves more balanced load assignment. In the condition of with the same number of work items, the algorithm in this article achieves lower the highest load of task participants, which reduces the overall load. Compared with that of the comparative algorithm, the time needed for all the tasks with the algorithm in this article is reduced by $27 \%$, improving the total task implementation efficiency.

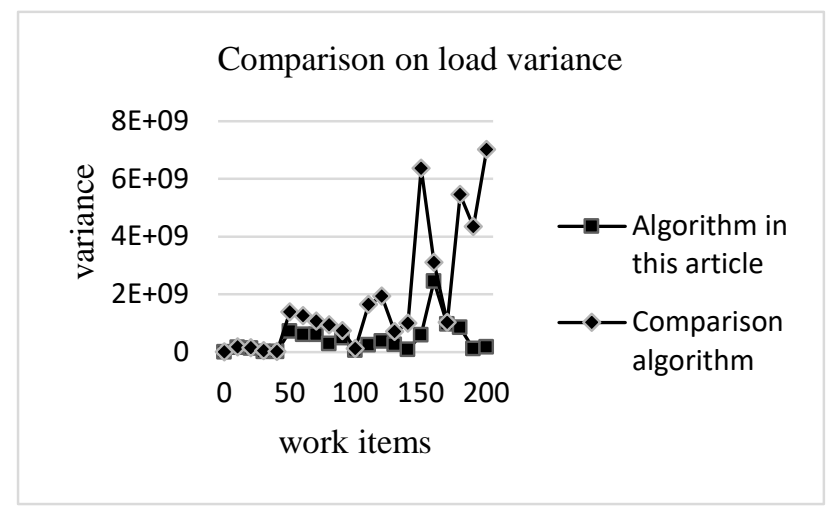

Fig. 3 Comparison on Variance of Algorithm Load 
According to the data shown in Fig. 3, the algorithm in this article can effectively control the load set variance of all the task participants in the system; compared with the comparative algorithm, the load set variance is significantly reduced, with maximum load balancing effect increase of $97.5 \%$ to the maximum, illustrating that the algorithm in this article can effectively reduce the difference on the loads between different task participants, playing a significant role in improving the balanced load between task participants.

\section{Conclusion}

This article proposes a traffic load based load balance task assignment strategy, which takes the traffic load of task participants of the mobile internet platform based workflow system into consideration. It calculates the needed traffic load by conducting line planning on task implementation locations in the task list of participants, which is then added with all the task implementation time in the task list, to ultimately acquire the final load value; besides, it takes the variance of the set composed by the load values of all the task participants as the standard for task assignment. Compared with other task assignment, this algorithm can significantly improve the load balancing effect of mobile internet platform based workflow system as well as the overall task implementation efficiency in the system.

\section{References}

[1] Zhu Yu. Research and Implementation of Workflow Engine [D]. University of Electronic Science and Technology of China, 2005.

[2] Liu Yi, Zhang Kan. Strategy for Workflow Task Assignment Based on Load Balance and Experiential Value[J]. Computer Engineering, 2009, 21: 57-59.

[3] Xiong Tianhong. Research on Workflow Engine Based on Task Equilibrium Assignment Algorithm [D]. Central South University, 2010.

[4] Jiang Jinsong, Yang Bo, Miao Zhimin, Zhu Baoshan. A Workflow Task Assignment Method based on the Properties of Task and User[J]. Computer Simulation, 2015, 12: 222-225+230.

[5] Li Chunfang, Xu Jianjun. Definition for business-oriented dynamic task allocation work model[J]. COMPUTER ENGINEERING AND DESIGN, 2014, 08: 2960-2964. 